\title{
El problema del seguimiento de reglas en Michael Oakeshott
}

\author{
The problem of rule-following in Michael Oakeshott
}

\author{
Juan Antonio González de Requena Farré \\ Universidad Austral de Chile \\ jgonzalez@spm.uach.cl
}

\begin{abstract}
Resumen
El papel de las reglas en nuestras prácticas normativas constituye un problema filosófico relevante, asociado principalmente con la filosofía de Wittgenstein, pero al cual Michael Oakeshott también ha tratado de dar respuesta. No en vano, algunos estudiosos han encontrado paralelismos entre sus concepciones de las prácticas humanas y del seguimiento de reglas. A través de una exégesis de la noción y usos de la regla en las obras de Oakeshott, este artículo pretende esclarecer el nexo entre seguimiento de reglas, y autorización normativa. De ese modo, será posible establecer la originalidad y especificidad del aporte de Oakeshott y dirimir la cuestión de las diferencias con la problematización wittgensteiniana del seguimiento de reglas.
\end{abstract}

Palabras clave: prácticas, seguimiento de reglas, normatividad, autoridad, asociación moral.

\begin{abstract}
The role of rules in our normative practices constitutes a relevant philosophical problema, mainly associated with Wittgenstein's philosophy, but to which Michael Oakeshott has also attempted to answer. Not surprisingly, some scholars have found parallels between their conceptions of human practices and rule-following. Through an exegesis of the notion and uses of the rule in Oakeshott's works, this article aims to clarify the link between rule-following and normative authorization. In this way, it will be possible to to decide the originality and specificity of Oakeshott's contribution and settle the question of the differences with the Wittgensteinian problematization of rule-following.
\end{abstract}

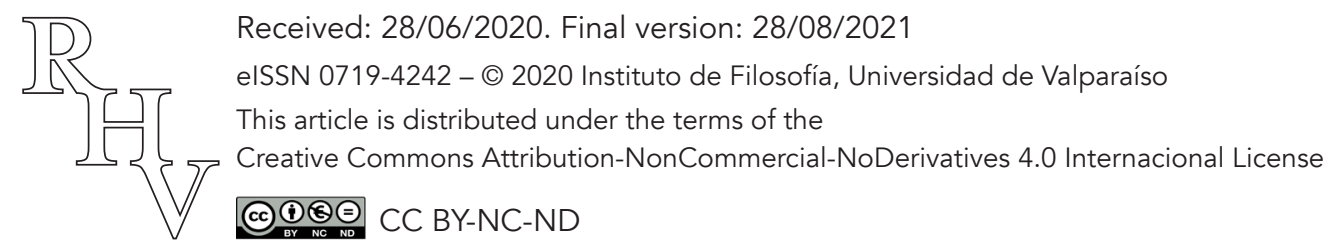


Keywords: practices, rule-following, normativity, authority, moral association.

\section{Introducción}

El problema filosófico del seguimiento de reglas parece relativamente contemporáneo (suele asociarse con cierta discusión wittgensteiniana), pero los discursos culturales y conceptualizaciones sociohistóricas centrados en las reglas tienen una larga data. En el discurso filosófico de la modernidad se registraron transformaciones relevantes en el léxico tradicional de las regulae, que básicamente se había empleado para referirse a las pautas de construcción y cánones arquitectónicos, las reglas del arte retórico y de la gramática, las reglas generales del derecho o los cánones de la vida religiosa. La moderna valoración intelectual del pensamiento metódico se tradujo en que los pioneros de la nueva ciencia tratasen de asegurar los derroteros de su investigación mediante la declaración de regulae para conducir el entendimiento. Las condiciones del pensar metódico, con su exigencia de reglas que pauten la investigación y permitan determinar regularidades estructurales objetivas, fueron proyectadas en el ámbito de los asuntos humanos por parte de las llamadas ciencias sociales, como evidencian las Reglas [regles] del método sociológico de Durkheim (2001).

Con el desarrollo e institucionalización académica de las ciencias sociales en el curso del siglo XX, el léxico de las reglas ocuparía un lugar decisivo en la comprensión del objeto de estudio de estas nuevas disciplinas, aunque las reglas se entendieron de diferentes modos en distintas tradiciones teóricas y metodológicas: como un patrón estandarizado de regulación funcional y cumplimiento de las actividades organizadas, que se expresa en técnicas, hábitos y preceptos dependientes de algún marco normativo (Malinowski 1985, 72-75); como una pauta formal de codificación y transformación estructural que regula los distintos juegos de comunicación cultural (estructuras de parentesco, de comunicación de bienes y servicios, o de mensajes) con independencia de la naturaleza de los participantes (Levi-Strauss 1987, 317-321); o bien como un esquema o estándar compartido de interpretación del sentido y de las expectativas intersubjetivas, que garantiza la inteligibilidad, consistencia, coherencia y la planificación en la comprensión común cotidiana, en tanto que resultado de las interacciones y solidaridades grupales (Garfinkel 2006). En todo caso, en las ciencias sociales contemporáneas también se ha cuestionado el canon de investigación basado en la observación externa desapegada y no comprometida, y se señaló tanto la ambigüedad de la noción de regla como los riesgos de confundir las distintas acepciones de la regla: regularidad objetiva condicionante, norma o principio consciente e intencionalmente producido, o modelos construidos por el investigador (Bourdieu 2000, 68).

También en el pensamiento jurídico contemporáneo se ha reproducido el léxico de las reglas (rule), para caracterizar la forma general de la ley y la normatividad del derecho. Esta autocomprensión del derecho como un sistema de reglas responde a la pretensión

Revista de Humanidades de Valparaíso, 2021, No 17, 289-310

(c) $(1)(9)$ CC BY-NC-ND 
El problema del seguimiento de reglas en Michael Oakeshott

Juan Antonio González de Requena Farré

positivista de construir científicamente un campo objetivamente diferenciado y una disciplina autónoma. Se trata de una vocación científica que responde en diferido al llamado de ciertas tendencias jurídicas modernas: la estatalización del derecho (la subsunción del derecho bajo la legislación, en el marco del centralismo político-jurídico del Estado), el absolutismo jurídico (un monismo riguroso de la legalidad, más allá del pluralismo normativo del Antiguo Régimen), el legalismo y la legolatría (el respeto a la forma pura de la ley como emanación del poder político soberano), y la codificación exhaustiva de las leyes en sistemas de normas generales y abstractas (la consagración del Código como forma de sistematización homogénea y simplificada de la legalidad) (Grossi 2008). En ese contexto, el positivismo jurídico contemporáneo ha sostenido que las reglas jurídicas son prescripciones humanas, aunque no se confunden con los imperativos morales y pueden investigarse autónomamente; desde esa perspectiva, el derecho constituiría un sistema cerrado de reglas generales, respaldadas por sanciones y dictadas por una instancia legis-

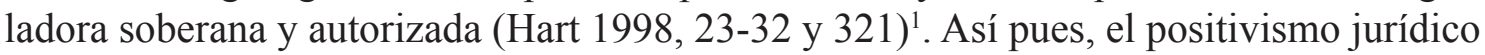
considera que el derecho consistiría en un conjunto de reglas válidas que acotan el campo de lo jurídico al sancionar ciertos comportamientos, de manera que solo cabría hablar de obligación si un caso cae bajo la regla válida correspondiente (Dworkin 1989, 65-66).

En la filosofía del derecho contemporánea, la conceptualización de la regla ha insistido frecuentemente en la autonomía normativa interna, de modo que autores como Raz han caracterizado las reglas prácticas como un tipo de razón opaca, independiente del contenido y autónoma, ya que no dependen de la deseabilidad o el valor de las acciones que justifican, sino de consideraciones normativas independientes; así pues, las reglas jurídicas serían razones protegidas válidas en la medida en que excluyen la acción por otras razones que la autonomía normativa de la regla (Raz 2013, 213-228). Desde una perspectiva decisional, Schauer (2004) ha caracterizado las reglas prescriptivas como razones para actuar, normativamente obligatorias, que exhiben cierta apertura y maleabilidad (ya que sus generalizaciones no siempre incluyen todos los casos y, a veces, incluyen más de lo debido), así como pueden arrojar resultados subóptimos respecto a la decisión particularista; además, la reglas se atrincherarían como generalizaciones y aportan justificaciones independientes de las razones subyacentes. En suma, el léxico de las reglas ha enmarcado gran parte del pensamiento jurídico contemporáneo, hasta el punto de introducir una visión del derecho sub specie regulae.

Ahora bien, en el panorama del pensamiento contemporáneo hallamos una posición filosófica en que convergen la problemática epistemológica del lugar de las reglas en la comprensión humana y, por otra parte, la problemática jurídica del papel de las reglas en la conformación del ordenamiento social y del Estado de derecho, a saber: la reflexión filosófica de Michael Oakeshott. Aunque la propuesta de Oakeshott posee un notable

\footnotetext{
${ }^{1}$ Como se apreciará en este trabajo, hay notables afinidades en el tratamiento de las reglas legales por parte de Oakeshott y Hart.
} 
El problema del seguimiento de reglas en Michael Oakeshott

Juan Antonio González de Requena Farré

potencial filosófico para concebir el problema del seguimiento de reglas de un modo más comprehensivo e integral, cabría pensar que no se han explorado en profundidad los aportes del pensador británico a una posible filosofía de las reglas. Pese a los esbozos de un abordaje de estas problemáticas en la bibliografía sobre Oakeshott, resulta preciso investigar más acuciosamente el modo en que su pensamiento permitiría replantear el problema del seguimiento de reglas. En este trabajo nos proponemos un suministrar una reconstrucción histórico-conceptual de los desplazamientos del vocabulario de las reglas en el pensamiento de Oakeshott. Adicionalmente, - - si bien no se pueden establecer influencias históricas, sino solo semejanzas intelectuales- en la segunda parte de este trabajo pretendemos remarcar la especificidad de la comprensión de las normas en Oakeshott mediante la comparación entre las perspectivas de Wittgenstein y Oakeshott en torno a la normatividad y el seguimiento de reglas.

La reflexión de Oakeshott representa un esfuerzo sistemático — que aúna la elucidación filosófica incondicional de las presuposiciones y la interpretación histórica circunstancial de los supuestos- para comprender los conceptos básicos que empleamos al pensar la actividad humana y la vida política (Nardin 2001, 4-14); en esa medida, nuestra propuesta de reconstrucción de la conceptualización de las reglas en el filósofo británico no fuerza su estilo intelectual. Además del interés teórico y bibliográfico inherente que pueda tener una obra capital del pensamiento contemporáneo como la de Oakeshott, la investigación de la problemática de las reglas constituye un relevante foco de reflexión transdisciplinaria, y hay un interés filosófico sustantivo de la problematización de las reglas en Oakeshott: frente a la crisis de legitimación del Estado de derecho, a la extensión de la razón instrumental y a la sequía normativa del discurso de las ciencias sociales, la filosofía política de Oakeshott involucra un decidido compromiso con la normatividad y autoridad de las reglas de la civilidad, pero también con el papel de las reglas en nuestra autocomprensión práctica cotidiana. En tiempos de desregulación generalizada y de estetización de la anomia, la autocomprensión normativa de la asociación civil hace razonable una nueva reflexión sobre el seguimiento de reglas en la vida pública.

\section{EI lenguaje de las reglas en Oakeshott}

Aunque el recurso al léxico de las reglas para llevar a cabo la articulación de la autocomprensión humana y el ordenamiento de la convivencia política constituye una apuesta crucial de la obra On human conduct (Oakeshott 2003), no siempre estuvo presente en la reflexión filosófica de Oakeshott. En ese sentido, la obra Experience and its modes (Oakeshott 1966) no concebía el razonamiento práctico y la acción moral como un asunto relacionado con el seguimiento, aplicación u observancia de alguna regla. Bajo la influencia del idealismo británico - la concepción monista u holista de la comprensión humana, la preferencia por la explicación histórica, la denegación de una realidad independiente de la experiencia, la concepción coherentista de la verdad o la diferenciación de las modali-

Revista de Humanidades de Valparaíso, 2021, No 17, 289-310

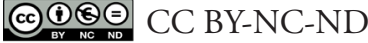


El problema del seguimiento de reglas en Michael Oakeshott

Juan Antonio González de Requena Farré

dades de experiencia-, Oakeshott trataba de dar cuenta de la experiencia humana en un marco neohegeliano, y asumía que la experiencia solo logra concebirse cabal y plenamente cuando se aprehende como una totalidad concreta y coherente, esto es filosóficamente ${ }^{2}$. Según Oakeshott, solo el pensamiento filosófico se tomaría en serio la superación de los confinamientos abstractos y unilaterales de la experiencia bajo los marcos intelectuales y modos de ideación de la ciencia, la historia o la práctica, que no consiguen agotar la totalidad concreta de la realidad. Desde esa perspectiva, cada modo de pensamiento constituye un mundo de experiencia acotado, y no es posible extrapolar las consideraciones y principios de homogeneidad de un ámbito a otro, sin incurrir en una falacia de ignoratio elenchi y, por ende, en la irrelevancia. Por ejemplo, el pensamiento histórico se torna irrelevante cuando intenta concebir la singularidad de los acontecimientos históricos como instancias de leyes generales o aplicaciones de alguna regla, como ocurre en algunas modalidades desafortunadas de filosofía de la historia que conciben leyes históricas generales por analogía con el pensamiento científico (limitado también a la consideración de generalizaciones hipotéticas que no agotan la realidad concreta) (Oakeshott 1966, 154). Desde la perspectiva de Oakeshott, también constituye un malentendido cierta representación práctica de la experiencia como si nuestras acciones estuviesen determinadas por reglas generales; en ese sentido, resultaría autocontradictoria y aberrante la concepción de la experiencia práctica como una simple aplicación de algún sistema de reglas o principios preexistentes, comparables a órdenes o consejos. Para Oakeshott $(1966,301)$, en el momento en que se asimilase la vida moral a la observancia de reglas, se perdería la característica distintiva de la experiencia práctica; es más, en Experience and its modes parece afirmarse cierta incompatibilidad entre la vida moral y la ley, entre la casuística y la sensibilidad moral.

Tampoco en Rationalism in politics and other essays (Oakeshott 1964), Oakeshott privilegia consistentemente el léxico de las reglas para dar cuenta de la autocomprensión humana y de la condición civil. De hecho, en el ensayo "Rationalism in politics" contenido en ese libro, las reglas se asocian a la formulación del conocimiento técnico, más que al saber práctico (que no puede formularse en reglas) o, incluso, al conocimiento científico (para el cual no bastan algunas reglas de observación y verificación). La actividad práctica no se dejaría reducir a la aplicación mecánica de un libreto de reglas técnicas, pues solo se adquiere mediante la participación en una forma tradicional de hacer las cosas y a través del contacto con quienes dominan ese idioma de actividad (Oakeshott 1964, 7-13). Del mismo modo, en varios ensayos de Rationalism in politics and other essays, se sostiene que los hábitos de conducta no se adquieren mediante la aplicación de reglas y preceptos, sino al vivir como la gente que se comporta habitualmente de cierta manera, en la medida en que comparte un idioma moral natal; por eso, solo se puede hablar fluidamente cuando nos olvidamos de las reglas del lenguaje. En otros ensayos del libro

\footnotetext{
${ }^{2}$ Sobre los supuestos filosóficos del idealismo británico, véase Boucher y Vincent (2012, 38-42).
}

Revista de Humanidades de Valparaíso, 2021, No 17, 289-310 
como "The tower of Babel" y "Rational conduct", Oakeshott reitera que la actividad humana no se deduce de principios premeditados ni de sistemas de reglas a priori, pues toda regla y principio sería tan solo una abreviatura abstracta de la actividad en que estamos situacionalmente involucrados; simplemente, compartimos cierto idioma de actividad y sus flujos de simpatía, de tal manera que participamos en una comprensión prácticamente adquirida y asumimos cierta fidelidad inmanente al despliegue de una actividad concreta. Así pues, la racionalidad de nuestras prácticas consistiría en la fidelidad a un idioma de actividad heredado — adquirido a través de la práctica-, el cual nos permite actuar apropiadamente según las circunstancias. Las tradiciones constituirían el modelo inherente a toda actividad humana; conformarían su coherencia sustancial y orientarían su flujo de simpatía, pero nunca bajo reglas abstractas, premeditadamente impuestas, sino mediante el ejercicio de habilidades adquiridas en tanto que hábitos familiares. Oakeshott (1964, 59-66 y 97-110) considera que las tradiciones compartidas de actividad se caracterizan por su elasticidad y su adaptabilidad a los matices de la situación, más que por su fijeza y rigidez; se conservan en el cambio habitual, como la frágil continuidad interna que estabiliza el devenir de los asuntos humanos. En ese sentido, Oakeshott (1964, 66-70) cuestiona la autocomprensión alternativa de la vida moral como aplicación reflexiva de criterios morales, ya sea a través de la búsqueda autoconsciente de ideales morales, ya sea como la observancia reflexiva de reglas y la interpretación casuística de las situaciones concretas en que se habría de aplicar la regla abstracta.

A pesar de lo que sostienen algunos intérpretes, Oakeshott no defiende unilateralmente la moralidad basada en los hábitos y flujos de simpatía, sino que pretende discernir filosóficamente dos formas ideales de la vida moral que en nuestras sociedades están inevitablemente entremezcladas, sin que resulte recomendable forzar alguno de estos ideales extremos: el del puro hábito o el de la reflexión pura (Oakeshott 1964, 59-70). De hecho, en algunos ensayos relacionados con la realización política de la actividad humana, Oakeshott parece reivindicar la importancia del reconocimiento de reglas en la conducción de la vida en común. En el ensayo "The political economy of freedom" contenido en Rationalism in politics and other essays, Oakeshott argumenta que el gobierno de la ley y la libertad política se sostienen en reglas establecidas, fijas y conocidas, que vinculan a gobernantes y gobernados, y limitan el empleo del poder (1964, 40-43). Otro ensayo del mismo libro, "On being conservative", destaca la importancia de las reglas para el gobierno de la ley; y es que, al establecer reglas generales en lugar de planes sustantivos de búsqueda de la verdad o la perfección, se dispone de arreglos fijos y familiares para que la gente tenga expectativas, pueda ordenar sus preferencias y evitar conflictos, y así persiga sus metas de modo opcional y reflexivo (Oakeshott 1964, 182-194).

En las conferencias de Harvard de 1958, publicadas póstumamente bajo el título Moral y política en la Europa moderna (Oakeshott 2008), se puede reconocer cierto énfasis o viraje kantiano en la reivindicación por parte de Oakeshott de las reglas como condiciones de la asociación moral no instrumental. Según el pensador británico, Kant habría 
El problema del seguimiento de reglas en Michael Oakeshott

Juan Antonio González de Requena Farré

formulado una versión genuinamente filosófica de la teoría política del individualismo. En términos generales, la política del individualismo expresaría cierta disposición moral de los individuos a autodeterminarse y escoger autónomamente; asume una concepción de la actividad de gobernar como la legislación autorizada, la administración de las reglas auténticas de la ley, el arbitraje legal y la armonización de los intereses individuales en un sistema de derechos comunes y con las condiciones asociadas al gobierno de la ley, que no sería una simple salvaguarda instrumental de los intereses sustantivos de los individuos. Para Oakeshott, la versión kantiana presupone el reconocimiento de la autonomía humana como irrenunciable disposición a autodeterminarse que ha de ser valorada en todo ser humano; además, Kant introduciría una concepción de la sociedad civil en tanto que asociación entre individuos que reconocen reglas externas no instrumentales a los intereses sustantivos individuales e iguales para todos, y así se obligan a respetar las condiciones constitutivas de su autoconducción. Frente a las asociaciones de tipo empresarial, que procuran instrumentalmente satisfacciones sustantivas o alguna concepción de un bien común, la asociación civil no tendría otro fin sino el reconocimiento de las condiciones formales de las reglas de la ley, sin las cuales no es posible la igual autonomía personal. En esa perspectiva kantiana, las reglas autorizadas son un presupuesto de la autonomía moral y de la asociación civil (Oakeshott 2008, 104-109).

En el ensayo "El aprendizaje y la enseñanza" (incluido en Oakeshott 2009), Oakeshott aborda la dimensión instruccional de las reglas, y se pegunta por el papel que desempeñan los distintos tipos de reglas en el conocimiento y en la enseñanza. El aprendizaje humano consistiría en un tipo de actividad inteligente y autónoma en virtud de la cual nos iniciamos en el conocimiento de toda una herencia de logros significativos, capacidades y modos de pensar. Oakeshott asocia el conocimiento humano no solo a lo que sabemos, sino a las capacidades que desplegamos al comprender o hacer algo con lo que sabemos; como conjunto de capacidades, el conocimiento involucra tanto información como juicio o discernimiento e, incluso, la expresión de un estilo propio. Los datos y hechos que conforman el componente informativo de nuestro saber resultan importantes porque aportarían reglas relacionadas con capacidades: en algunos casos, las reglas se dan a través de informaciones que se han de conocer para actuar (como en el caso de una fórmula química o una receta de cocina); en otros casos, las reglas aportan criterios (cierta gramática presupuesta en el uso de un idioma) para saber si una acción se realiza de modo correcto y detectar errores, aunque no resulte imprescindible conocerlas; también contamos con otro tipo de regla que suministra los principios explicativos o la lógica subyacente para dar cuenta de una acción y sus pautas, aun cuando no sea conocimiento constitutivo de la acción ni de sus criterios de desempeño (Oakeshott 2009, 68-79).

En todo caso, Oakeshott considera que el contenido informativo del conocimiento, así como el repertorio de reglas que expresan lo que sabemos y nuestras capacidades (incluso si no siempre las seguimos expresamente), no agota el aspecto implícito o tácito de lo que sabemos. El saber cómo, es decir, el discernimiento o juicio que nos permite actuar y 
comprender circunstancialmente, no se reduce al saber qué informacional. Según Oakeshott, las reglas generales (las cuales se limitan a marcar una disyuntiva de acción) solo nos capacitan para una explicación parcial e imperfecta de lo que hacemos, pues resulta necesario el juicio no codificable en reglas y la capacidad de interpretar y seleccionar actuaciones cuando no hay reglas precisas. Por otro lado, la concreción singular de nuestras capacidades se expresa como un estilo propio para hablar el idioma de alguna actividad humana, explorando opcionalmente los espacios de libertad que dejan las reglas. Esta importancia del discernimiento y del estilo personal no rige únicamente en las relaciones prácticas, en la medida en que las reglas morales y legales o las máximas prudenciales no agotan nuestra comprensión práctica; también en el caso de los lenguajes de la ciencia, la filosofía, la historia o el arte se presuponen reglas de comprensión, pero solo se puede hablar competentemente esos idiomas si somos capaces de expresarnos significativamente de un modo no determinado por las reglas. Por eso, la educación no puede limitarse a la instrucción informativa y al aspecto instruccional de las reglas: pasa por la transmisión de discernimiento o la capacidad de pensar personalmente y adquirir un estilo, sin el cual no hay conocimiento humano logrado (Oakeshott 2009, 80-91).

El léxico de las reglas vuelve a desempeñar un papel fundamental en los dos primeros ensayos que componen On human conduct (Oakeshott 2003). El primer ensayo del libro, "On the theoretical understanding of human conduct", asocia la comprensión humana a una indagación sin fin, en que todo entendimiento de la actividad inter homines resulta condicional a alguna plataforma de comprensión y remite a cierta práctica, esto es, a conjuntos de reglas, usos y consideraciones que, al ser empleados más que aplicados, califican adverbialmente las elecciones y acciones de los agentes. Nuestra comprensión de los acontecimientos tendría lugar en términos de un reconocimiento de caracteres o tipos ideales compuestos de características ideales, y existirían distintas plataformas de comprensión condicional que permiten relacionar los acontecimientos a través de sus rasgos comunes o circunstancias, al formular teoremas o leyes como presupuestos explicativos. En ese sentido, la filosofía se perfila como una aventura intelectual incondicional que explora el carácter condicional de los presupuestos de nuestra comprensión. Oakeshott distingue dos categorías de acontecimientos: por un lado, los procesos que presuponen algún tipo de regularidad, condición causal o ley explicativa (por ejemplo, los teoremas de la mecánica), y, por otro lado, aquellos acontecimientos que exhiben autocomprensión y constituyen la respuesta inteligente de un agente reflexivo, libre y persuasivo, frente a su situación comprendida mediante la participación en alguna práctica humana. Cada práctica humana "puede identificarse como un conjunto de consideraciones, maneras, usos, observancias, costumbres, estándares, máximas canónicas, principios, reglas y oficios, que especifican procedimientos convenientes o denotan obligaciones o deberes que se relacionan con acciones y enunciados humanos" (Oakeshott 2003, 55).

En la práctica moral, que nos permite interactuar con otros agentes, autoexplorarnos y escenificarnos, pueden reconocerse construcciones familiares o reglas de comportamien- 
to que compendian las condiciones de deseabilidad y especifican obligaciones; se trata de abreviaturas abstractas de las consideraciones de la práctica y estipulan condiciones generales para escoger, pero no pueden determinar rígidamente lo que ha de hacerse. En ese sentido, el repertorio de reglas que constituye una práctica se asemeja a un lenguaje natal coloquial o idioma vernáculo, cuya gramática se emplea y actualiza cotidianamente, sin posible identificación con la demostración de teoremas, la aplicación abstracta de preceptos generales o la prescripción de actuaciones sustantivas. Estamos, pues, ante una versión más elaborada de las intimaciones o sugerencias de la tradición, a que Oakeshott apelaba en obras anteriores como guía legítima de la actividad humana. Por eso, Oakeshott insiste nuevamente en que la comprensión de una práctica y las artes de la acción humana no se reducen al seguimiento de un libro de reglas que prescriban actuaciones determinadas, sino que presuponen el dominio práctico tácito de la gramática de nuestro lenguaje moral; esto es, involucran el empleo de sus recursos y condiciones contingentes, así como el reconocimiento de la autoridad y utilidad de sus reglas idiomáticas (Oakeshott 2003, 60-68).

El segundo ensayo contenido en On human conduct, titulado "On the civil condition", caracteriza la relación de civilidad como un tipo de vinculación distinta de la satisfacción de deseos personales o necesidades compartidas, en la medida en que se trata de una relación en términos de reglas no instrumentales de conducta, que no prescriben acciones sustantivas, sino condiciones a suscribir cuando se actúa y escoge. Oakeshott retoma el planteamiento de que las prácticas son solo condiciones comprendidas y suscritas (usos y costumbres, máximas generales o bien reglas) que califican las actuaciones, pero no determinan ejecuciones o patrones de comportamiento; como expresiones de actividad inteligente pueden reglamentarse, pero sus reglas siempre son reinventadas al emplearlas, del mismo modo que un lenguaje solo se aprende y comprende al usarlo significativamente e inventar enunciados. Oakeshott indaga la comprensión de la interacción humana en el lenguaje moral natal aportado por una práctica y la articula con la relación de civilidad, en tanto que asociación moral no instrumental, exclusivamente formulada en términos de las reglas y normas legales que preservan la libertad de los agentes. Desde esa perspectiva, las reglas que sustentan la relación de civilidad se diferencian de los procedimientos e instrucciones de una asociación empresarial, que persigue instrumentalmente algún bien sustantivo; pero también se distinguen de las reglas de un juego, que presupone algún tipo de acuerdo y compromiso común entre los jugadores. Las reglas de la civilidad permiten que perdure la asociación civil entre aquellos agentes que no comparten una meta común o un compromiso compartido, e incorporan un tipo de obligación civil sustentada únicamente en el reconocimiento de las reglas como reglas, es decir, en el reconocimiento de su autoridad, más que en la simple aprobación de las condiciones normativas.

En On human conduct, particularmente en el ensayo "On the civil condition", encontramos una caracterización general de las reglas que dan forma a las prácticas humanas como un idioma moral vernáculo: las reglas especifican condiciones adverbiales com- 
El problema del seguimiento de reglas en Michael Oakeshott

Juan Antonio González de Requena Farré

prendidas que se han de suscribir y observar al escoger; tienen forma normativa y presuponen el reconocimiento de la autoridad; no son consejos $u$ órdenes circunstanciales ni directrices administrativas; no son argumentos para justificar teóricamente o predecir empíricamente acciones o comportamientos regulares, pues solo apelan a la suscripción y reconocimiento de ciertas condiciones normativas; determinan una jurisdicción, pero no interpelan a alguien en particular; se trata de prescripciones permanentes que no se agotan en una actuación determinada; no imponen acciones o enunciaciones específicas, sino que estipulan condiciones que han de ser reconocidas; además, las reglas cobran sentido en el contexto de alguna práctica, o sea, de un conjunto de condiciones comprendidas y un lenguaje propio, y no requieren la aprobación o desaprobación de sus condiciones ni el cálculo de sus consecuencias (Oakeshott 2003,124-127).

Posteriormente, en el ensayo "El concepto de rule of law" (incluido en Oakeshott 2013), se presentarían de nuevo los postulados del Estado de derecho y se reiteraría la caracterización de ese modo ideal de asociación moral implicado en las prácticas y relaciones humanas que tienen por única condición las reglas reconocidas como leyes auténticas y autorizadas. Oakeshott insiste en que las reglas de la ley no son leyes de la naturaleza o regularidades con capacidad explicativa y predictiva: se trata de artefactos e invenciones que declaran las condiciones reconocidas y no instrumentales de cierto tipo de relación humana, distinta de la asociación transaccional (que persigue la utilidad mutua y consecuencias sustantivas) y de las asociaciones comunitarias (que promueven una meta sustantiva común, como necesidades, deseos o intereses colectivos). Oakeshott se sirve de la analogía del juego, para aclarar el tipo de relación procedimental y no instrumental de las asociaciones morales basadas exclusivamente en el reconocimiento de reglas: en el juego, aparte de los preceptos estratégicos y prescripciones instrumentales para ganar la partida, se presuponen ciertas reglas que solo formulan ciertas condiciones formales implicadas en el reconocimiento del propio juego; no son pautas para ejercer el poder ni para obtener ventaja de los competidores, pues solo son las condiciones arbitrarias de participación en una actividad autónoma; no dan órdenes específicas ni determinan actuaciones sustantivas, sino solo declaran las obligaciones recíprocas constitutivas del juego; en ese sentido, se crearon mediante la deliberación, resultan modificables y no requieren la consulta permanente de un manual de instrucciones. No obstante, las reglas de un juego se caracterizan por una ambivalencia que no se da en una asociación exclusivamente moral: "exhiben el carácter dual de todas las reglas genuinas: pueden ser consideradas en función de su autenticidad o en función de las condiciones que prescriben" (Oakeshott 2013, 126).

Como se argumenta en "El concepto de rule of law" (Oakeshott 2013), las reglas auténticas no son como las máximas, instrucciones o consejos, pues no emplean un lenguaje prudencial relativo a las consecuencias sustantivas de las acciones y a su conveniencia. Al tener una jurisdicción no restringida a ciertos actores y ocasiones (pues solo prescriben condiciones formales para cualquier actuación), tampoco son como las órdenes dirigidas a personas específicas para que respondan obedeciendo; la validez de las reglas se aso-

Revista de Humanidades de Valparaíso, 2021, No 17, 289-310

(c) $(1)(9)$ CC BY-NC-ND 
cia al reconocimiento de su autenticidad o autoridad, y no a la sujeción a una orden y al poder sancionador. Por otra parte, las reglas auténticas no constituirían simples criterios o estándares para establecer lo correcto y lo incorrecto, lo aprobable y lo desaprobado, ya que solo prescriben condiciones formales reconocidas y obligaciones autorizadas para nuestras actuaciones autónomas. Ahora bien, según Oakeshott, el modelo ideal de una asociación moral, sustentada exclusivamente en el reconocimiento de reglas, aún resulta ambivalente, ya que no diferencia la autenticidad de la regla (su legalidad) y, por otro lado, su rectitud o justicia. Esa ambigüedad solo se resolvería bajo el gobierno de la ley y en el Estado de derecho, cuando se distinguen las funciones del legislador soberano (capaz de promulgar leyes autorizadas) y el dictamen judicial de los tribunales (que hacen justicia en términos de verificar, bajo ciertas condiciones procesales formales, el cumplimiento o incumplimiento de obligaciones legales auténticas en el caso de acciones o enunciaciones contingentes). En ese marco de la rule of law, las reglas de la ley solo expresan "prescripciones inevitablemente indeterminadas de obligaciones adverbiales de naturaleza general" (Oakeshott 2013, 140).

Los especialistas en Oakeshott concuerdan en la relevancia de las reglas no instrumentales para las prácticas morales de una asociación civil. Por ejemplo, Paul Franco (1990) considera que, en On human conduct, la asociación civil no se confunde con una práctica instrumental basada en algún propósito sustantivo, ya que constituye una relación formal o práctica moral, con la particularidad de que es un tipo de asociación moral completamente constituida y articulada por reglas. Ahora bien, las reglas reconocidas en la asociación civil no serían simples compendios o códigos, pues se trata de un lenguaje de reglas hablado como una tradición viviente y una práctica moral. Las reglas auténticas que harían posible la asociación civil como relación en términos no instrumentales se caracterizarían por constituir enunciados autorizados y no teoremas; no son enunciados argumentativos demostrables, y su validez no depende de su razonabilidad o aprobación. Además, las reglas no se asimilarían a órdenes específicas, ya que tienen una forma abstracta y una jurisdicción general. Por otra parte, las reglas no prescriben enunciados sustantivos, sino solo consideraciones formales o de procedimiento, que han de suscribirse al actuar autónomamente. Por último, las reglas auténticas resultarían reconocidas en términos del respeto de las obligaciones y de su autoridad, pero no en virtud de las consecuencias de obedecerlas o no. Desde esa perspectiva, concluye Franco, los ciudadanos vinculados por la asociación civil solo se relacionan en términos del reconocimiento de reglas auténticas autorizadas (Franco 1990, 182-185).

Especialistas como Terry Nardin (2001) también reconocen en la obra de Oakeshott un intento filosófico de distinguir la forma de relación humana propia de la civilidad como una modalidad ideal de gobierno de la ley, en que las personas se vinculan mediante reglas reconocidas, autorizadas y no instrumentales. De ese modo, no habría que confundir esa rule of law con la mezcla de reglas, mandatos, órdenes y directivas que se encuentran contingentemente en un sistema legal concreto. De hecho, las leyes de un Estado concreto 
pueden exhibir características de reglas constitutivas no instrumentales y rasgos de reglas instrumentales para el logro de algún propósito sustantivo. Para Nardin, lo que Oakeshott plantea es que cada Estado concreto sería una construcción ambivalente: combina una comprensión instrumental de los propósitos sustantivos y las directivas administrativas $\mathrm{y}$, por otro lado, una comprensión no instrumental de la asociación civil como práctica basada en el reconocimiento de reglas auténticas y autorizadas. No en vano, las reglas no instrumentales implicarían el reconocimiento de su autoridad como único criterio exclusivo de su autenticidad, al margen de consideraciones sobre su eficacia para lograr alguna meta sustantiva. Según Nardin, aunque los sistemas legales concretos puedan mezclar aspectos instrumentales y no instrumentales, en Oakeshott se plantearía teóricamente una diferencia categorial de género y modalidad entre el modo de asociación en términos de reglas no instrumentales y, por otra parte, la asociación instrumental al logro de propósitos sustantivos; bajo ningún concepto han de confundirse (Nardin 2001, 196-203).

\section{La cuestión de las afinidades entre Oakeshott y Wittgenstein}

El planteamiento filosófico de Oakeshott no es el único enfoque teórico que ha reivindicado el lugar central de las reglas en la comprensión de la conducta humana y, más específicamente, ha apelado a la relevancia del problema del seguimiento de reglas, a la hora de dar cuenta de la normatividad de las prácticas sociales. En su libro Ciencia social y filosofía (Winch 1990), Peter Winch defendió la importancia del análisis epistemológico de la comprensión humana, para explicitar la constitución de las relaciones sociales y de la sociedad, y asumió que la problematización wittgensteiniana del seguimiento de reglas permitía clarificar la conducta significativa y los empleos del lenguaje, siempre en el marco social de determinados contextos de actividad y formas de vida. Para Winch, seguir una regla (como ocurre al reconocer una definición o aplicar una fórmula matemática) no consiste simplemente en hacer lo mismo o seguir un procedimiento idéntico, ya que solo desde la regla se concibe la identidad relevante (y las diferencias); tampoco pasa por decidir intuitiva o discrecionalmente en cada caso, puesto que la aplicación de la regla implica cierta regularidad. Para distinguir la actuación de quien sigue una regla y la de quien no la aplica, no basta con apelar a una fórmula, ya que siempre se podrían interpretar las acciones como aplicación de alguna fórmula alternativa: resulta preciso considerar las respuestas de otros individuos y los acuerdos interpersonales que hacen posible reconocer las acciones incorrectas y corregir las aplicaciones erróneas. La conducta significativa ha de entenderse, pues, como seguimiento de reglas y aplicación reflexiva de criterios para distinguir los modos correctos y erróneos de actuar. Esa normatividad de las prácticas sociales se concebiría cabalmente sobre el trasfondo de una comprensión compartida y de la familiaridad con determinado contexto e instituciones sociales (Winch 1990, 29-64).

David Bloor también ha considerado decisiva la discusión sobre el seguimiento de reglas, para hacerse cargo de las prácticas sociales y de la relación entre teoría y práctica

Revista de Humanidades de Valparaíso, 2021, No 17, 289-310

(c) $(9)(9)$ CC BY-NC-ND 
en la vida social. Según Bloor (2001), la problematización wittgensteiniana de las reglas permite remarcar la primacía de la práctica sobre la teoría, pese a que el seguimiento de reglas parece ejemplificar la precedencia del contenido proposicional sobre las aplicaciones. Para reivindicar la práctica implicada en el seguimiento de reglas, Wittgenstein habría señalado el carácter ilusorio o mítico de la total autonomía ideal de las reglas, como si estas encarrilaran misteriosamente cualquier aplicación posible; solo se pueden entender estas ilusiones como efecto de las prácticas sociales, y la comprensión del seguimiento de reglas debería naturalizarse en el marco de las formas de vida social. Para Bloor, la normatividad implicada en el seguimiento de reglas derivaría del consenso entre los participantes y del hábito ciego; en ese sentido, el seguimiento de reglas involucra cierta conciencia de las instituciones relevantes y de la participación en la actividad social (en vez de la mera conformidad conductual), pero se trataría de una conciencia rutinaria desplegada sobre un trasfondo familiar (y no de interpretaciones adicionales, proposiciones factuales o explicaciones teóricas), la cual se adquiere a través de un entrenamiento social. Las reglas existirían como instituciones y costumbres sociales, y resultarían socialmente constituidas mediante procesos autorreferenciales de autoinstitución de la realidad social, al invocar reglas para glosar ciertas actividades y dar cuenta verbalmente de lo que hacemos; así, el seguimiento de reglas consistiría en la participación en prácticas institucionales relevantes y normativamente autocomprendidas (Bloor 1997; véase también 2001). Frente a las interpretaciones individualistas del seguimiento de reglas, que presuponen cierto determinismo del significado conceptual y una misteriosa fuerza impulsora inherente a la normatividad de la regla, Bloor considera preciso atender a las condiciones sociales que informan nuestras disposiciones a actuar y suministran estándares de corrección. Así pues, la comprensión del seguimiento de reglas resultaría inseparable del modo en que aprendemos y enseñamos reglas a partir de ejemplos específicos y de ratificaciones consensuales de nuestros modos de actuar: los estándares normativos implicados en el seguimiento de reglas provienen del consenso de los participantes, y se mantienen a través del control social y la sanción colectiva sobre el desempeño del individuo (Bloor 1997, 27-42).

Winch y Bloor no solo comparten una interpretación comunitaria del seguimiento de reglas, sino que además ambos trazan cierto paralelismo entre el abordaje de las reglas en Wittgenstein y en el pensador británico Michael Oakeshott. Desde una perspectiva wittgensteiniana, Winch comparte el rechazo de Oakeshott hacia la sobrevaloración racionalista de la autonomía y carácter apriorístico de la inteligencia humana, como si la racionalidad de la acción humana derivase de un intelecto autosuficiente. Y es que en las actividades humanas se da una constante acción recíproca de razones y pasiones. Las actividades sociales no se deducen formalmente de algún análisis de fines y medios, sino que presuponen una familiaridad con contextos y tradiciones; de ese modo, la actividad humana no se puede reducir a un conjunto explícito de preceptos abstractos. Para Winch, Oakeshott generaliza a toda actividad humana la posibilidad de describirla como hábito 
El problema del seguimiento de reglas en Michael Oakeshott

Juan Antonio González de Requena Farré

o costumbre, que no requeriría de regla ni de reflexión explícita. Winch replica que la posibilidad de formular explícitamente una regla no es decisiva para establecer que se está siguiendo alguna regla, pues lo importante es si se puede distinguir entre modos correctos e incorrectos de actuar y, por ende, si se aplica un criterio (aunque no se formule), tal como ocurre al responder a las correcciones de un maestro y al poder proseguir como se me ha enseñado. En suma, el seguimiento de reglas no equivaldría al hábito o propensión a continuar haciendo lo mismo según las insinuaciones de la tradición (como plantearía Oakeshott); en la actividad social marcan la diferencia la aplicación reflexiva de criterios y la interpretación de las situaciones nuevas. Así, la reflexividad y la posibilidad de comprender formas de actuación alternativas serían inseparables de la conducta significativa, aunque actuar no consista una simple derivación desde principios reflexivos a priori (Winch 1990, 53-64).

Para Bloor (2001), Wittgenstein y Oakeshott comparten una oposición al racionalismo, entendido como la afirmación de la primacía de la teoría sobre la práctica. En ese sentido, tanto Wittgenstein como Oakeshott representarían cierto conservadurismo, esto es, la defensa de la práctica situada y concreta sobre las pretensiones abstractas de la teoría. El mérito de Wittgenstein habría consistido precisamente en describir los matices de la relación entre pensamiento y acción, para elucidar la relación conflictiva entre racionalismo teórico y conservadurismo de la práctica; su principal logro habría sido redescribir la cuestión aparentemente racional y teórica del seguimiento de reglas (crucial en lógica y matemática), de manera que se convierte en un paradigma de primacía de la práctica sobre la teoría. Al fin y al cabo, según Bloor, el análisis wittgensteiniano permite concluir que las reglas constituyen instituciones sociales, de manera que seguir una regla sería participar en una institución social cuyos modos de conducirse se enseñan y aprenden colectivamente. En fin, tanto Wittgenstein como Oakeshott proporcionarían una descripción no racionalista de la actividad y el pensamiento humanos, centrada en las prácticas sociales contextuales (Bloor 2001, 103-104).

Tras una lectura cuidadosa del tratamiento de las reglas la obra de Oakeshott, los paralelismos que algunos estudiosos de Wittgenstein han establecido entre ambas posiciones se tornan bastante discutibles. En ese sentido, el argumento crítico de Winch respecto al carácter irreflexivo de las prácticas normativas en Oakeshott solo se sostiene en una lectura parcial de algunos ensayos recogidos en Rationalism in politics and other essays (aquellos en que se privilegia el seguimiento de las sugerencias de la tradición por sobre la aplicación de reglas), pero no considera un amplio conjunto de textos en que se reivindica el lugar de las reglas en la articulación de las prácticas humanas y en el despliegue de la convivencia bajo la forma de la asociación moral y del Estado de derecho (particularmente, On human conduct). Por otra parte, la interpretación conservadora que Bloor hace de Wittgenstein y Oakeshott — como si en ambos autores se afirmase el predominio de la práctica sobre la teoría- depende demasiado de la exégesis comunitaria del seguimiento de reglas (por ejemplo, la propuesta por Kripke 2006; véase también Malcolm 
El problema del seguimiento de reglas en Michael Oakeshott

Juan Antonio González de Requena Farré

1989) y, por tanto, presupone que seguir reglas consiste en participar consensualmente en prácticas e instituciones colectivas que se autoconstituyen sustantivamente como formas de vida en común. Sin embargo, ni en Wittgenstein ni en Oakeshott resulta obvia la interpretación comunitaria del seguimiento de reglas. En Wittgenstein, la interpretación comunitaria plantea problemas a la comprensión de la relación interna entre la regla y su aplicación; además, desdibuja los estándares normativos contenidos en las reglas, sin los cuales no se podría diferenciar la actuación conforme a una regla - aunque fuese consensual- y el seguimiento de reglas implicadas en la comprensión en alguna práctica, así como tampoco se lograría distinguir entre el creer que se sigue una regla y el seguirla efectivamente (Baker y Hacker 1984, 71-80). En Oakeshott, el planteamiento comunitario suscita dificultades respecto al carácter formal y procedimental de las reglas genuinas, que no se pueden asimilar a órdenes, máximas prácticas o consejos instrumentales para el logro de los propósitos sustantivos de una comunidad, ya que solo prescriben condiciones generales para la actuación autónoma (Nardin 2001, 201-202).

Algunos estudiosos de la obra de Oakeshott también han intentado establecer algún tipo de paralelismo con el pensamiento de Wittgenstein. Por ejemplo, Isaacs (2006) ha señalado que Wittgenstein y Oakeshott participan de cierto idealismo: ambos consideran que la filosofía solo tiene que explicar la gramática o los postulados de lo que ya sabemos y los modos de experiencia que forjamos condicionalmente, pero no puede hacerse cargo de una realidad desconocida ni asumir hechos absolutamente dados. Para Isaacs, ambos autores comparten cierta posición no fundacional y antiesencialista, así como un cuestionamiento del énfasis racionalista en estructuras últimas o absolutas. Wittgenstein introduce la noción de formas de vida en las cuales se despliegan fluidamente nuestros juegos de lenguaje mediante la participación en sus prácticas; Oakeshott se serviría del léxico de las tradiciones para caracterizar el trasfondo flexible de la comprensión humana, consistente en voces e idiomas de actuación. Wittgenstein se sirve de los juegos de lenguaje para describir los contextos de empleo, prácticas o regiones lingüísticas; Oakeshott se refiere a ciertos modos de experiencia, voces o prácticas con un lenguaje propio. Según Isaacs, una coincidencia importante entre ambos autores concierne a las reglas: tanto Wittgenstein como Oakeshott asociarían la comprensión al seguimiento de reglas implicadas en las prácticas humanas y en la experiencia común, y los dos considerarían que las reglas constituyen normas convencionales de actuación expresadas al obrar (Isaacs 2006, 164-169).

Por su parte, Luke Plotica (2015) considera que la visión de las prácticas humanas como modos de autocomprensión y conceptualización articulados en idiomas característicos concordaría con el énfasis del último Wittgenstein en la gramática de los juegos de lenguaje como marco de nuestras prácticas. Plotica recoge los argumentos de algunos intérpretes sobre la convergencia intelectual de Wittgenstein y Oakeshott: ambos compartirían cierta actitud o estilo filosófico opuesto al racionalismo, y privilegiarían la perspectiva de un actor situado cuya comprensión depende de capacidades compartidas, reglas comunes, condiciones sociales, relaciones intersubjetivas y horizontes de sentido

Revista de Humanidades de Valparaíso, 2021, No 17, 289-310

(c) $(9)(9)$ CC BY-NC-ND 
El problema del seguimiento de reglas en Michael Oakeshott

Juan Antonio González de Requena Farré

colectivos; los dos darían cuenta de la acción humana en virtud de la participación comunitaria en prácticas o juegos de lenguaje estructurados mediante el seguimiento de reglas, y se preocuparían del modo en que las reglas articulan nuestras actividades, lenguajes y prácticas significativas; en ambos casos, el estudio de la acción humana exigiría la consideración de los marcos prácticos de empleo del lenguaje, juegos de lenguaje o modos idiomáticos.

Plotica (2015) se muestra crítico con cierta interpretación conservadora de Wittgenstein y Oakeshott, de acuerdo con la cual ambos pensadores privilegiarían la práctica sobre la teoría (o bien las reglas comunitarias, sobre la actuación individual); como si las personas, al actuar, solo pudieran reproducir su acuerdo tácito con convenciones y tradiciones colectivas. El argumento básico de la interpretación conservadora de Wittgenstein y Oakeshott se sostiene en ciertos supuestos: la acción humana está enmarcada constitutivamente por el empleo del lenguaje; el empleo del lenguaje consiste en prácticas convencionales e intersubjetivas, así como en el seguimiento de reglas convencionales de una comunidad; de ese modo, la corrección en el seguimiento de reglas consistiría en la concordancia con la costumbre comunitaria, y la actuación individual estaría determinada por la capacidad de adecuarse a las convenciones comunitarias (Plotica 2015, 24-25). Según Plotica, en Wittgenstein y Oakeshott resulta concebible la comprensión y la actuación humana como un ejercicio de iniciativa y agencia personales - no exento de reflexión y crítica-, sin que ello implique desconocer las condiciones sociales e intersubjetivas de las prácticas e instituciones; no en vano, la actuación personal y crítica da vitalidad a nuestras prácticas significativas $\mathrm{y}$, así, configura constitutivamente nuestras formas de vida (Plotica 2015, 15-37).

Si sopesamos los argumentos formulados por los estudiosos de Wittgenstein y Oakeshott respecto a cierto paralelismo entre sus obras, resulta razonable concluir que en ambos pensadores se cuestiona la ambición racionalista de deducir todas las formas de actividad y conocimiento humanos a partir de un código explícito de reglas abstractas; además, los dos comparten una perspectiva de la comprensión centrada en las prácticas significativas estructuradas y en el seguimiento de reglas convencionales a través de nuestra participación en juegos de lenguaje e instituciones compartidas. No obstante, conviene matizar el paralelismo entre las concepciones del seguimiento de reglas en Wittgenstein y Oakesho$\mathrm{tt}$, mediante una consideración más detallada del papel de las reglas en nuestras prácticas significativas. En ese sentido, más que un escepticismo respecto al seguimiento de reglas en las prácticas humanas, ambos pensadores ponen de manifiesto un cuestionamiento de cierta concepción rígida y exhaustiva del seguimiento de reglas, como si se emplease el lenguaje bajo las reglas estrictas de un cálculo, o bien existiese un libreto a priori de reglas abstractas a partir de las cuales se puedan deducir nuestras prácticas contingentes y eventuales actuaciones. Al fin y al cabo, Wittgenstein insistió en que no todo contexto de actividad presupone la formulación explícita de reglas como un catálogo a aplicar, ya que también se puede participar en un juego de lenguaje prácticamente, al observar el

Revista de Humanidades de Valparaíso, 2021, No 17, 289-310

(c) $(1)(9)$ CC BY-NC-ND 
El problema del seguimiento de reglas en Michael Oakeshott

Juan Antonio González de Requena Farré

desempeño ajeno (1984, 39-42; véase también 1988, §§ 53-54). Por su parte, Oakeshott nos recordó que las sutiles insinuaciones de nuestras tradiciones no se dejan deducir de reglas abstractas, y sostuvo que existen formas de comprensión competente y expresión significativa más allá de las reglas. Los dos pensadores concuerdan en el reconocimiento de que hay capacidades tácitas y trasfondos no explicitables involucrados en el seguimiento de reglas: ya se trate del dominio ciego de las técnicas presupuestas en nuestras prácticas y la familiaridad implícita con las formas de vida que sustentan nuestros juegos de lenguaje (Wittgenstein 1988, $\S \S 197-219$; véase también 1987, 289-297); ya se trate de las insinuaciones de nuestras tradiciones, de la gramática inherente a nuestros idiomas de actividad, del saber cómo característico del discernimiento, o bien del estilo singular que caracteriza una comprensión lograda (en el caso de Oakeshott).

Asimismo, tanto Wittgenstein como Oakeshott se toman muy en serio el aspecto instruccional de las reglas. De acuerdo a la interpretación de Meredith Williams (1999), Wittgenstein concibe el seguimiento de reglas y la actividad normativa como una iniciación en una práctica social estructurada de la comunidad, y la objetividad del seguimiento de reglas radicaría en la posibilidad de distinguir las aplicaciones correctas e incorrectas en virtud de la normatividad de las reglas, así como en la constricción que la necesidad de las reglas introduce en las actuaciones de los participantes de una práctica comunitaria. Según Williams, la problematización wittgensteiniana del seguimiento de reglas se aleja de la solución intelectualista que entiende su aplicación como una interpretación o justificación adicional de las reglas; no en vano, el modo en que nos hacemos competentes en el seguimiento de reglas y adquirimos su comprensión consiste en el adiestramiento en las técnicas habituales de una práctica social. Por eso, la normatividad de las reglas se basaría en la continuidad de la concordancia comunitaria, y la necesidad o carácter constrictivo de las reglas se fundaría en un proceso de aculturación social en el contexto estructurado de las interacciones de una comunidad. Así, para Williams, la obediencia ciega a la necesidad de las reglas consistiría en atenerse a cómo han de ser las cosas en una forma de vida que permite reconocer las desviaciones y aciertos a partir de cierta concordancia común y del desempeño de quienes dominan sus técnicas. El aprendizaje resultaría decisivo para obtener la maestría en aquellas técnicas que constituyen el trasfondo del seguimiento de alguna regla: la comprensión de una regla no solo se logra al dominar sus aplicaciones, sino también al describir cómo se aprendió la regla, esto es, cómo el maestro en alguna práctica se relaciona con un aprendiz y le aporta cierto saber cómo no declarativo, al ejemplificar las actuaciones correctas y corregir las incorrectas, mientras el aprendiz se somete ciegamente a la autoridad del maestro en la práctica compartida, sin que se precise una ulterior justificación del trasfondo de nuestra forma de vida (Williams 1999, 157-187).

De manera análoga, Oakeshott (2009) considera que la enseñanza resulta decisiva para la iniciación de los individuos históricos en los logros heredados de la humanidad, y concibe el conocimiento aprendido como un conjunto de capacidades que nos permiten em-

Revista de Humanidades de Valparaíso, 2021, No 17, 289-310

(c) $(9)(9)$ CC BY-NC-ND 


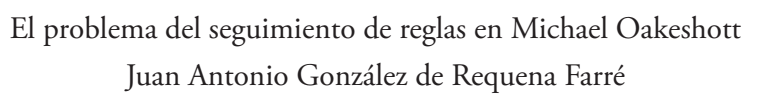

plear cuanto sabemos, mediante actuaciones y comprensiones. En ese sentido, las reglas hacen posible transformar la información declarativa en pautas de actuación, y nos suministran criterios de comprensión e, incluso, principios explicativos; pero, para Oakeshott, además de suministrar instrucción en el seguimiento de reglas, la educación concierne a la transmisión de la capacidad de discernimiento y a la adquisición de un estilo propio, más allá de las reglas. Eso sí, según Oakeshott, en esa labor de transmisión, el maestro no adiestra o condiciona al aprendiz, sino que solo le brinda ejemplos, le permite contactarse con un modelo de desempeño a imitar y le comunica un estilo de comprensión y actuación virtuosa, para que el alumno logre desplegar su exploración intelectual autónoma (Oakeshott 2009).

Para la comprensión de las prácticas normativas, resulta decisivo otro aspecto en el cual coinciden Wittgenstein y Oakeshott, esto es, la distinción de los diferentes tipos de reglas incluso en un mismo juego de lenguaje o idioma práctico. En el pensamiento de Oakeshott se distinguen diferentes tipos de regla en contextos educativos y prácticos: las reglas informacionales (que hacen posible disponer de instrucciones para el empleo de algunos datos), las reglas gramaticales (presupuestas al hablar un idioma de actividad y al reconocer sus usos correctos), o bien las reglas explicativas (que dan cuenta de alguna actividad humana); por otro lado, las reglas instrumentales o estratégicas (que indican metas sustantivas y orientan la actividad propositiva), o bien las reglas no instrumentales (que solo prescriben las condiciones formales generales de la actuación autónoma en una asociación moral). A través de sus descripciones de distintos juegos de lenguaje, Wittgenstein nos ha familiarizado con reglas tan distintas como las pautas básicas de reconocimiento, las señales indicativas, las tablas de equivalencia, las instrucciones operacionales o las reglas complejas de inferencia.

En algunos estudiosos y continuadores del pensamiento de Wittgenstein, encontramos descripciones sumamente matizadas de la diversidad de reglas implicadas en los contextos de actividad humana. Así, en su lógica de las normas, Georg Von Wright (1970, 21-35) formuló la diferencia entre las reglas de un juego o las reglas gramaticales, las normas o regulaciones prescriptivas, las directrices técnicas, así como ciertos casos intermedios: los hábitos regulares, las normas morales y las reglas que establecen patrones ideales. En su filosofía de los actos de habla, John Searle (1990, 42-46) distinguió entre las reglas regulativas (imperativos condicionales que orientan una actividad preexistente y lógicamente independiente de las reglas) y las reglas constitutivas (que dan forma a actividades cuya existencia depende lógicamente de esas reglas, al establecer que algo cuenta como movimiento del juego). Por su parte, Stanley Cavell (2003, 407-409) consideró que existen al menos cuatro tipos de reglas diferentes en una práctica: las reglas definitorias o constitutivas de la práctica, las reglas normativas, los principios estratégicos y las máximas pragmáticas. En fin, cualquier descripción rigurosa de nuestras prácticas 
normativas debiera contemplar este pluralismo de las reglas, en vez de deslumbrarse con el espejismo de que existen sistemas consistentes y exhaustivos de reglas estrictas en las actividades humanas.

\section{A modo de conclusión: la especificidad de la comprensión normativa del segui- miento de reglas en Oakeshott}

Como hemos argumentado, en el pensamiento de Oakeshott, el papel que las reglas desempeñan en las prácticas compartidas y en la comprensión humana solo se puede concebir adecuadamente en el contexto de una actividad de teorización filosófica para la cual las reglas se perfilan como un idioma natal o un lenguaje coloquial constitutivo de las prácticas humanas; así concebidas, las reglas especifican condiciones formales de nuestras elecciones, tienen un trasfondo normativo y presuponen el reconocimiento de su autoridad. Ciertamente, Oakeshott parece compartir con Wittgenstein no solo el cuestionamiento de la pretensión racionalista de deducir la realidad humana de una codificación explícita de reglas abstractas, sino también una comprensión centrada en las prácticas significativas estructuradas normativa e institucionalmente. Ahora bien, una comparación lograda entre los pensamientos de Wittgenstein y Oakeshott respecto al papel de las reglas pasa por establecer sus divergencias.

Sin duda, hay una diferencia de énfasis en el abordaje del seguimiento de reglas por parte de ambos autores, que permite entender la especificidad de la comprensión normativa de Oakeshott: aunque los dos emplean el paradigma del juego, para describir las reglas de empleo contextual del lenguaje y clarificar las condiciones de la participación en prácticas significativas, en Wittgenstein predominan las descripciones esquemáticas y ahistóricas de contextos de seguimiento de reglas en los cuales tiene lugar, por ejemplo, el reconocimiento ostensivo de un contenido conceptual, la representación de pautas de significado, la transformación de unas representaciones simbólicas en otras, o bien la aplicación operacional de patrones de inferencia a partir de expresiones simbólicas (Wittgenstein 1984, 124-136; véase también 1987, 329-337, y 1988, §§ 185-237). Pero, en esos contextos, las reglas no existirían en sí o simplemente como formulaciones simbólicas, sino que solo tendrían vigencia como instrumentos empleados en actividades normativas, al cumplir un papel en la orientación de la acción, en la explicación, justificación, evaluación y enseñanza (Baker y Hacker 2009, 50-55). Sin embargo, en Oakeshott se enfatiza la dimensión intersubjetiva de las reglas como constituyentes de ciertos modos de relación humana históricamente contingentes y, eventualmente, como la base de una asociación moral entre personas, cuando los individuos autónomos reconocen las reglas auténticas en tanto que reglas no instrumentales, y sostienen su convivencia en la pura normatividad y autoridad de las reglas.

En ese sentido, los paradigmas de normatividad preferidos en el pensamiento wittgensteiniano corresponden a representaciones abstractas e idealizadas como tablas, es-

Revista de Humanidades de Valparaíso, 2021, No 17, 289-310 


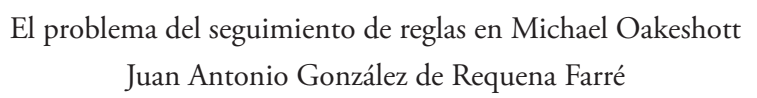

quemas, indicaciones e instrucciones, mientras que Oakeshott considera que la forma más lograda de seguimiento de reglas se da concretamente en los sistemas legales y en el modelo histórico ideal del Estado de derecho. En ese marco de las reglas de la ley, no basta con apelar al adiestramiento instruccional y la obediencia ciega a los criterios inmanentes de nuestras prácticas comunitarias (como podría pensarse con Wittgenstein). Tampoco es suficiente argumentar que, ante las paradojas derivadas de un planteamiento abstracto y desapegado del problema, el trasfondo del seguimiento de reglas consiste en un modo de comprensión implícito en nuestra actividad, que nos suministraría un sentido práctico inarticulado, encarnado en ciertas disposiciones corporales y pautas dialógicas de interacción (Taylor 1997, 221-238). Y es que la clave de la normatividad y obligatoriedad de las reglas auténticas de una asociación moral radica en la autoridad reconocida y en el papel autorizador de las reglas, tanto en el momento de su promulgación, como en el momento de dictaminar sus aplicaciones y hacerlas valer (como sostiene Oakeshott 2003, 130-154). En suma, la simple descripción analítica de ciertos contextos abstractos de seguimiento de reglas no consigue dar cuenta de la normatividad y obligatoriedad de nuestras más sutiles prácticas normativas, históricamente concretadas en comprensiones institucionales contingentes: aquellas en que las reglas se reconocen y siguen en tanto que reglas, solo en virtud de su autoridad normativa, y no de su aceptación consensual, de alguna visión comprehensiva del bien común o de algún propósito comunitario sustantivo. He ahí una enseñanza para los recuentos de las prácticas normativas en ciencias sociales.

\section{Agradecimientos}

Artículo vinculado al proyecto Fondecyt Regular no 1190030, investigación financiada por ANID.

\section{Referencias bibliográficas}

Baker, Gordon P., Hacker, Peter M. S (1984). Scepticism, Rules and Language. Oxford: Blackwell.

Baker, Gordon P., Hacker, Peter M. S. (2009). Wittgenstein: Rules, Grammar and Necessity. Essays and Exegesis of \$\$185-242. Oxford: Wiley-Blackwell.

Bloor, David (1997). Wittgenstein, rules and institutions. London: Routledge.

Bloor, D. (2001). Wittgenstein and the priority of practice. En T. R. Schatzki, K. Knorr Cetina y E. von Savigny (eds.), The Practice Turn in Contemporary Theory, pp. 103-114. London: Routledge.

Boucher, David, Vincent, Andrew (2012). British idealism: a guide for the perplexed. London: Continuum International Publishing. 
Bourdieu, Pierre (2000). Cosas dichas. Barcelona: Gedisa.

Cavell, Stanley (2003). Reivindicaciones de la razón. Madrid: Síntesis.

Durkheim, Emile (2001). Las reglas del método sociológico. México, D.F.: Fondo de Cultura Económica.

Dworkin, Ronald (1989). Los derechos en serio. Barcelona: Ariel.

Franco, Paul (1990). The Political Philosophy of Michael Oakeshott. London: Yale University Press.

Garfinkel, Harold (2006). Estudios en Etnometodología. Barcelona: Anthropos.

Grossi, Paolo (2008). Europa y el derecho. Barcelona: Crítica.

Hart, Herbert L. A. (1998). El concepto de derecho. Buenos Aires: Abeledo-Perrot.

Isaacs, Stuart (2006). The politics and philosophy of Michael Oakeshott. New York: Routledge.

Kripke, Saul (2006). Wittgenstein: a propósito de reglas y lenguaje privado. Una exposición elemental. Madrid: Tecnos.

Levi-Strauss, Claude (1987). Antropología estructural. Barcelona: Paidós.

Malcolm, N. (1989). Wittgenstein on Language and Rules. Philosophy, 64(247), 5-28.

Malinowski, Bronislaw (1985). Una teoría cientifica de la cultura. Madrid: Sarpe.

Nardin, Terry (2001). The philosophy of Michael Oakeshott. Pennsylvania: The Pennsylvania State University Press.

Oakeshott, Michael (1964). Rationalism in politics and other essays. London: Methuen.

Oakeshott, Michael (1966). Experience and its modes. Cambridge: Cambridge University Press.

Oakeshott, Michael (2003). On human conduct. Oxford: Clarendon Press.

Oakeshott, Michael (2008). Moral y política en la Europa moderna. Madrid: Síntesis.

Oakeshott, Michael (2009). La voz del aprendizaje liberal. Buenos Aires: Katz.

Oakeshott, Michael (2013). Sobre la historia y otros ensayos. Buenos Aires: Katz.

Plotica, Luke P. (2015). Michael Oakeshott and the conversation of modern political thought. Albany, New York: State University of New York Press.

Raz, Joseph (2013). Entre la autoridad y la interpretación. Sobre la teoría del derecho y la razón práctica. Madrid: Marcial Pons.

Searle, John (1990). Actos de habla. Madrid: Cátedra. 
Schauer, Frederick (2004). Las reglas en juego. Madrid: Marcial Pons.

Taylor, Charles (1997). Argumentos filosóficos. Barcelona: Paidós.

Von Wright, Georg H. (1970). Norma y acción. Una investigación lógica. Madrid: Tecnos.

Williams, Meredith (1999). Wittgenstein, mind, and meaning: toward a social conception of mind. London: Routledge.

Winch, Peter (1990). Ciencia social y filosofía. Buenos Aires: Amorrortu.

Wittgenstein, Ludwig (1984). Los cuadernos azul y marrón. Madrid: Tecnos.

Wittgenstein, Ludwig (1987). Observaciones sobre los fundamentos de la matemática. Madrid: Alianza Editorial.

Wittgenstein, Ludwig (1988). Investigaciones filosóficas. Barcelona: Crítica. 